\title{
NONSEPARATING ALMOST CONTINUOUS RETRACTS OF $I^{n}$
}

\author{
HARVEY ROSEN
}

\begin{abstract}
Compact almost continuous retracts of $I^{n}(n \geqslant 2)$ do not separate $E^{n}$. Some other results that hold for continuous functions are also shown to hold for almost continuous functions. A result in [5] giving sufficient conditions for a set to be an almost continuous retract of $I^{n}$ is examined further, and a method of constructing some almost continuous retracts of $I^{n}$ is given.
\end{abstract}

1. Introduction. Almost continuous or connectivity functions sometimes obey the same type theorems that continuous functions obey. Sometimes they do not. For example, no connectivity function $f: S^{n} \rightarrow S^{n-1}$ commutes with the antipodal map [3], but some almost continuous function does [4]. Also, every connectivity or almost continuous retract of $I^{n}$ has the fixed point property $[2,9]$.

Theorem 3.16 in [2] states that every connectivity retract of an $n$-cell in $E^{n}(n \geqslant 2)$ is a nonseparating subcontinuum of $E^{n}$. Similarly, we can show that the closure of an almost continuous retract $Y$ of an $n$-cell in $E^{n}(n \geqslant 2)$ is a subcontinuum of $E^{n}$ and that $Y$ cannot separate $E^{n}$ if $Y$ is compact. Because it can apply to any compact almost continuous retract $Y$ of $I^{n}$ relative to $I^{2 n}$ which might not be an almost continuous retract relative to $I^{n} \times Y$, the preceding result is an extension of Theorem 4 in [5]. Some other results of algebraic topology are shown to hold for almost continuous functions. We also show that some of the sufficient conditions for a set $M$ to be an almost continuous retract of $I^{n}$ can be dropped from Theorem 1 of $[5]$.

In the last section, some almost continuous retracts of $I^{n}(n \geqslant 2)$ are constructed by removing infinitely many half-open $n$-cells from $I^{n}$.

2. Definitions and examples. A function $f: X \rightarrow Y$ is called almost continuous relative to $X \times Y$ if every neighborhood $U$ of (the graph of) $f$ contains some continuous function $g: X \rightarrow Y$. One familiar almost continuous function is any derivative $F^{\prime}:[a, b] \rightarrow R$. We say that a closed subset $K$ of $X \times Y$ is a blocking set of $X \times Y$ if $K$ misses some function from $X$ into $Y$ but meets every continuous function from $X$ into $Y$. A function $f: X \rightarrow Y$ is almost continuous relative to $X \times Z$ if $Y \subset Z$ and every neighborhood $U$ of $f$ in $X \times Z$ contains a continuous function $g: X \rightarrow Z$. It turns out that almost continuity of $f$ relative to $X \times Y$ implies almost continuity of $f$ relative to $X \times Z$, but not vice versa. A subcontinuum $Y$ of $X$ is an almost continuous retract of $X$ relative to $X \times Z$ if $Y \subset Z$ and there is an almost continuous function $f: X \rightarrow Y$ relative to $X \times Z$ which is the identity on $Y$. If $X=Z=I^{n}$, we

Received by the editors March 10, 1982 and, in revised form, July 11, 1983.

1980 Mathematics Subject Classification. Primary 54C08, 54C15; Secondary 54H25.

Key words and phrases. Almost continuous retracts of $I^{n}$, blocking sets, fixed point property. 
shall say simply that $Y$ is an almost continuous retract of $I^{n}$ or that $f$ is an almost continuous retraction of $I^{n}$ onto $Y$. When

$$
Y=\left\{\left(x, \sin \frac{1}{x+1}\right):-1<x \leqslant 1\right\} \cup\{(-1,0)\}
$$

and $I=[-1,1]$, it is not hard to verify that vertical projection of $I^{2}$ onto $Y$ is an example of an almost continuous retraction $f: I^{2} \rightarrow Y$ relative to $I^{2} \times Y$ with a nowhere dense graph in $I^{2} \times Y$.

For a subset $K$ of $I^{n} \times Y$, we let $p_{x}(K)$ denote the projection of $K$ into the first coordinate space $I^{n}$.

3. Nonseparating property. We show how to modify the proof of Theorem 3.16 of [2] to obtain the following result.

THEOREM 1. If $Y$ is a compact almost continuous retract of $I^{n}(n \geqslant 2)$, then $Y$ does not separate $E^{n}$.

Proof. Let $f: I^{n} \rightarrow Y$ be an almost continuous retraction. If $Y$ separates $E^{n}$, there exists an essential continuous function $g$ of $Y$ onto $S^{n-1}$, the boundary of the unit $n$-ball $D^{n}$ in $E^{n}$. Since $D^{n}$ is an AR, there is a continuous extension $G: I^{n} \rightarrow D^{n}$ of $g$. Of course, $G\left(I^{n}\right) \not \subset S^{n-1}$; however, $G f: I^{n} \rightarrow D^{n}$ is almost continuous relative to $I^{n} \times D^{n}$ [9] and $G f\left(I^{n}\right) \subset S^{n-1}$. Let $B$ be the open $n$-ball in $E^{n}$ with center at the origin $O$ and radius 2, and let $A$ be the open annulus $B-\{O\}$. Define

$$
U=\left[\left(I^{n}-Y\right) \times A\right] \cup\{(p, q): p \in Y, q \in A, \text { and } d(q, g(p))<1\}
$$

where $d$ denotes the Euclidean metric on $E^{n}$. Since $U$ is an open set containing $G f$, there is a continuous function $h: I^{n} \rightarrow D^{n}$ such that $h \subset U$. Let $\phi$ be a retraction of $A$ onto $S^{n-1}$ such that $d(x, \phi(x))<1$ for all $x$ in $A$. Then $\phi h\left(I^{n}\right) \subset S^{n-1}$ because $h\left(I^{n}\right) \subset A$. If $p \in Y$, then $d(g(p), h(p))<1$ and $d(h(p), \phi h(p))<1$. Therefore $d(g(p), \phi h(p))<2$. Since $g(p)$ and $\phi h(p)$ are not antipodal points of $S^{n-1}, \phi h \mid Y$ is homotopic to $g$. It follows that $\phi h: I^{n} \rightarrow S^{n-1}$ is essential, a contradiction.

COROLlaRY 1. There exists no almost continuous retraction of $I^{n}$ onto its boundary.

CoRollary 2. Let $S^{n}$ be the boundary of the unit $(n+1)$-ball $D^{n+1} . S^{n}$ is not contractible by means of an almost continuous homotopy relative to $S^{n} \times I \times D^{n+1}$.

Proof. Assume there is an almost continuous function $H: S^{n} \times I \rightarrow S^{n}$ relative to $S^{n} \times I \times D^{n+1}$ such that $H(x,-1)=x$ for all $x \in S^{n}$ and $H\left(S^{n}, 1\right)=\{q\} . S^{n} \times\{1\}$ bounds an $(n+1)$-cell $D_{1}=D^{n+1} \times\{1\}$ such that $D_{2}=\left(S^{n} \times I\right) \cup D_{1}$ is an $(n+1)$-cell. We can extend $H$ to a function $F: D_{2} \rightarrow S^{n}$ so that $F\left(D_{1}\right)=\{q\}$. If $F$ were not almost continuous relative to $D_{2} \times D^{n+1}$, then there would be a neighborhood $U$ of $F$ in $D_{2} \times D^{n+1}$ that contains no continuous function from $D_{2}$ into $D^{n+1}$. Since $\left(D_{2} \times D^{n+1}\right)-U$ misses $D_{1} \times\{q\}$, there exists an open $(n+1)$-cell neighborhood $V$ of $q$ in $D^{n+1}$ such that $D_{1} \times V \subset U$. The neighborhood $U-\left[\operatorname{Bd}\left(D_{1}\right) \times\right.$ $\left.\left(D^{n+1}-V\right)\right]$ of $H$ contains a continuous function $h: S^{n} \times I \rightarrow S^{n}$. Because $h\left(\mathrm{Bd} D_{1}\right) \subset V, h$ can be extended to a continuous function $g: D_{2} \rightarrow D^{n+1}$ with $g \subset U$. On account of this contradiction, $F: D_{2} \rightarrow S^{n}$ is almost continuous relative to $D_{2} \times D^{n+1}$. But according to Corollary 1 , this is impossible. 
Other results that hold for continuous functions sometimes hold for almost continuous functions. Although we pointed out in the introduction that the BorsukUlam Theorem does not hold for almost continuous functions [4], the theorem about the nonexistence of a continuous nonzero distribution $f: S^{2 n} \rightarrow E^{2 n}$ of tangent vectors on $S^{2 n}$ still does. For, if $f: S^{2 n} \rightarrow E^{2 n}$ is an almost continuous nonzero discontinuous distribution of tangent vectors, then $p f: S^{2 n} \rightarrow S^{2 n}$ is almost continuous where $p(x)=x /|x|$ [9]. Therefore, there is a point $x_{0}$ which $p f$ either leaves fixed or maps to its antipode; otherwise, the neighborhood $S^{2 n} \times S^{2 n}-\{(x, y)$ : $y= \pm x\}$ of $p f$ would contain a continuous function $g: S^{2 n} \rightarrow S^{2 n}$, an impossibility. Then $x_{0}$ is not orthogonal to $f\left(x_{0}\right)$.

Example. Does $Y$ have to be compact in Theorem 1? Of course, it is possible for $\bar{Y}$ to separate $E^{n}$ if $Y$ is a noncompact almost continuous retract of $I^{n}$; an example, $M_{3}$, was given in [5] of such a set $Y . M_{3}$ is a point on a circle along with a spiral beginning at the center and limiting on the circle. Theorem 1 of [5] gives sufficient conditions for a set $M$ like $M_{3}$ to be an almost continuous retract of $I^{2}$ (and thus have the fixed point property); however, a gap in the proof of Theorem 1 occurs when it is stated that $p_{x}(K)-T$ is perfect, where $p_{x}(K)$ is the projection into $I^{n}$ of a minimal blocking set $K$ of $I^{n} \times M$ that misses some function $f: I^{n} \rightarrow M$ constructed there and $T$ is the finite set of all isolated points of $p_{x}(K)$. For, $M$ might not be compact and $p_{x}(K)$ might not be closed. The referee noticed that a gap seems to occur also in the proof of Lemma 2 in [5] when the dense arc component $A_{0}$ of $M$ is apparently assumed to be open in $M$. (However, the spiral $A_{0}$ in the example $M_{3}$ mentioned above is open in $M_{3}$.) In correcting Theorem 1 of [5] with Theorem 2 below, we show we can omit from the hypothesis the conditions that $M$ contains no simple closed curve and that the arc components of $M$ except for $A_{0}$ are nowhere dense in $M$ and finite in number.

The following lemma is a generalization of Theorem 2 in [7].

LEMMA 1. If $A_{0}$ is an arcwise connected subset of $E^{n}$, then for each minimal blocking set $K_{0}$ of $I^{n} \times A_{0}, p_{x}\left(K_{0}\right)$ is a nondegenerate connected set.

Proof. It is obvious that $p_{x}\left(K_{0}\right)$ contains more than one point. Suppose $p_{x}\left(K_{0}\right)$ is not connected and $p_{x}\left(K_{0}\right)=A \cup B$ with $A$ and $B$ separated. Since $I^{n}$ is completely normal, there exist in $I^{n}$ disjoint open sets $U \supset A$ and $V \supset B$. No points of $p_{x}\left(K_{0}\right)$ are in $\operatorname{Bd}(U)$ or $\operatorname{Bd}(V) . K_{0} \cap\left(U \times A_{0}\right)$ and $K_{0} \cap\left(V \times A_{0}\right)$ are closed in $I^{n} \times A_{0}$, and neither can be a blocking set of $I^{n} \times A_{0}$. Therefore, there exist continuous functions $g_{1}, g_{2}: I^{n} \rightarrow A_{0}$ such that $p_{x}\left(K_{0} \cap g_{1}\right) \subset V$ and $p_{x}\left(K_{0} \cap g_{2}\right) \subset U$. For each positive integer $i$, let $U_{i}$ be the open $i^{-1}$-neighborhood of $\operatorname{Bd}(U)$ in $I^{n}$, and let $V_{i}$ be the open $i^{-1}$-neighborhood of $\operatorname{Bd}(V)$ in $I^{n}$. Let $B_{0}$ be an arc in $A_{0}$ that meets both $g_{1}\left(I^{n}\right)$ and $g_{2}\left(I^{n}\right)$, and let $C=g_{1}\left(I^{n}\right) \cup B_{0} \cup g_{2}\left(I^{n}\right)$. It follows from the regular neighborhood collaring theorem [8, p. 36] that $g_{i}=g_{1}\left|\left(\bar{U}-U_{i}\right) \cup g_{2}\right|\left(\bar{V}-V_{i}\right)$ can be extended to a continuous function $G_{i}: I^{n} \rightarrow C$ so that $G_{i}\left(I^{n}-N_{i}\right)$ is a point for some regular neighborhood $N_{i}$ in $U \cup V$ of a compact polyhedron containing $\left(\bar{U}-U_{i}\right) \cup\left(\bar{V}-V_{i}\right)$. For each $i, G_{i}$ meets $K_{0}$ in some point of $\left(U_{i} \cup V_{i}\right) \times C$. Since $K_{0}$ is closed and $C$ is compact, $K_{0}$ must meet $[\operatorname{Bd}(U) \cup \operatorname{Bd}(V)] \times C$, a contradiction. 
THEOREM 2. Let $M$ be a subset of $I^{n}$ with an arc component $A_{0}$ that is dense in $M$. Suppose there exists a function $f_{0}: \bar{M} \rightarrow M$ which is the identity on $M$ and there exists $a$ sequence of continuous functions $g_{1}, g_{2}, \ldots$ such that $g_{i}: I^{n} \rightarrow A_{0}$ and such that if $P_{1}$, $P_{2}, \ldots$ is a sequence of points of $\bar{M}$ converging to $P$, then $g_{1}\left(P_{1}\right), g_{2}\left(P_{2}\right), \ldots$ converges to $f_{0}(P)$. Then $M$ is an almost continuous retract of $I^{n}$ relative to $I^{n} \times M$.

Proof. We begin the proof as in [5]. Let $\theta$ be the set of all closed subsets $L$ of $I^{n} \times M$ for which $p_{x}(L)$ has $c$-many points not in $\bar{M}$. Using transfinite induction, we can define a function $f: I^{n} \rightarrow M$ such that $f \mid \bar{M}=f_{0}$ and $f$ meets each $L$ in $\theta$. We need only show that $f$ is almost continuous relative to $I^{n} \times M$. Assume $f$ is not. Then by Theorem 3 of [5], there exists a minimal blocking set $K$ of $I^{n} \times M$ that misses $f$.

We show that for each $x$ in $I^{n},\{x\} \times A_{0}$ is not contained in $K \cap(\{x\} \times M)$. Assume that some $\{x\} \times A_{0}$ is contained in $K \cap(\{x\} \times M)$. Since $M \subset \overline{A_{0}}$ and $K$ is closed in $I^{n} \times M$, then $\{x\} \times M=\{x\} \times\left(\overline{A_{0}} \cap M\right) \subset K \cap(\{x\} \times M)$. Therefore, $(x, f(x)) \in K$ in contradiction to the fact that $K \cap f=\varnothing$. Then $\{x\} \times A_{0} \nsubseteq K \cap$ $(\{x\} \times M)$ for all $x \in I^{n}$.

For each $x \in I^{n}$, choose a point $(x, y) \in\left(\{x\} \times A_{0}\right)-(K \cap(\{x\} \times M))$. This defines a function $h: I^{n} \rightarrow A_{0}$ such that $y=h(x)$ and $K \cap\left(I^{n} \times A_{0}\right)$ misses $h$. If $g$ : $I^{n} \rightarrow A_{0}$ is a continuous function, then $g$ meets the blocking set $K$ of $I^{n} \times M$ and hence meets $K \cap\left(I^{n} \times A_{0}\right)$. This shows that $K \cap\left(I^{n} \times A_{0}\right)$ is a blocking set of $I^{n} \times A_{0}$. According to Theorem 3 in [5], since $h: I^{n} \rightarrow A_{0}$ is not almost continuous and $I^{n}$ is compact, there exists a minimal blocking set $K_{0}$ of $I^{n} \times A_{0}$ that misses $h$. Then $K_{0}$ meets each $g_{i}$ in some point $\left(P_{i}, g_{i}\left(P_{i}\right)\right)$.

Without loss of generality, we can suppose $K_{0}$ is contained in $K \cap\left(I^{n} \times A_{0}\right)$. For, in the proof of Theorem 3 in [5], one could have considered a chain of biocking sets contained in a prescribed blocking set (such as $K \cap\left(I^{n} \times A_{0}\right)$ here). Then Zorn's lemma would, as in [5], give a minimal blocking set now contained in the prescribed one.

By Lemma $1, p_{x}\left(K_{0}\right)$ is a nondegenerate connected set. Then $p_{x}\left(K_{0}\right) \subset \bar{M}$, and so each $P_{i}$ must lie in $\bar{M}$. Otherwise, if $p_{x}\left(K_{0}\right) \nsubseteq \bar{M}$, then $p_{x}\left(K_{0}\right)$ and hence $p_{x}(K)$ would have $c$-many points not in $\bar{M}$. This is impossible because $f$ was constructed to meet each closed subset $L$ of $I^{n} \times M$ whenever $p_{x}(L)$ has $c$-many points not in $\bar{M}$, yet $f \cap K=\varnothing$.

Continuing as in the proof in [5], we may assume that $P_{1}, P_{2}, \ldots$ converges to some point $P$. According to the hypothesis, $g_{1}\left(P_{1}\right), g_{2}\left(P_{2}\right), \ldots$ converges to $f_{0}(P)=$ $f(P)$. Therefore $(P, f(P))$ is in the closed set $K$, a contradiction. This shows that $f$ must be an almost continuous retraction of $I^{n}$ onto $M$ relative to $I^{n} \times M$.

4. Construction of some almost continuous retracts of $I^{n}, n \geqslant 2$. Kellum has noticed that, according to [6], if $M_{1} \subset I^{2}$ is Knaster's indecomposable continuum with one endpoint, then $M_{1}$ is an almost continuous retract of $I^{2}$. In [1], Garrett shows that

$$
M_{2}=\mathrm{Cl}\left\{\left(x, \sin \frac{1}{x}\right):-1 \leqslant x \leqslant 1, x \neq 0\right\}
$$


is an almost continuous retract of $I^{2}$. Both of these facts also come from the next general result because $M_{1}$ and $M_{2}$ can be obtained in the same way as the following continuum $M$. Here, interior and boundary of a cell are its combinatorial ones.

TheOREM 3. Suppose $D_{1}, D_{2}, D_{3}, \ldots$ are topological $n$-cells in $I^{n}$ with pairwise disjoint interiors such that each $\mathrm{Bd} D_{i}$ is the union of $(n-1)$-cells $E_{i}$ and $B_{i}$ with $B_{i}=\operatorname{Bd}\left(D_{i}\right)-\operatorname{Int}\left(E_{i}\right)$ and $E_{i} \subset \operatorname{Bd} I^{n}$. Let $M=I^{n}-\cup_{i=1}^{\infty}\left(D_{i}-B_{i}\right)$. Then $M$ is an almost continuous retract of $I^{n}$.

Proof. Define $r: I^{n} \rightarrow M$ so that $r$ is the identity on $M$ and for $i=1,2,3, \ldots, r \mid D_{i}$ is a (continuous) retraction of $D_{i}$ onto $B_{i}$. Possibly, $r$ might be discontinuous. We shall show $r$ is an almost continuous retraction of $I^{n}$ onto $M$.

Let $\varepsilon>0$, and let $U$ be the $\varepsilon$-neighborhood of $r$ in $I^{2 n}$ and $V$ be the $\varepsilon / 2$-neighborhood of $r \mid M$ in $U$. It follows there is a neighborhood $N$ of $r \mid M$ in $V$ with the property that if $(z, y) \in N$, then $d(z, y)=\bar{d}((z, z),(z, y))<\varepsilon / 2$, where $d$ and $\bar{d}$ denote the Euclidean metrics on $E^{n}$ and $E^{2 n}$ respectively. There exists a positive integer $m$ such that for all $i>m, D_{i}$ is contained in the projection $p_{x}(N)$ of $N$ into the first coordinate space $I^{n}$ of $I^{n} \times I^{n}$. Define the continuous function $g: I^{n} \rightarrow I^{n}$ that is the identity on $M$ and on $D_{i}$ for all $i>m$ and so that $g=r$ on $D_{i}$ for $i=1,2, \ldots, m$. Now suppose $i>m$ and $z \in D_{i}$. Since $z \in p_{x}(N)$, there is some $y$ such that $(z, y) \in N$. Therefore $\bar{d}((z, z),(z, y))<\varepsilon / 2$, and there is some $w \in M$ such that $\bar{d}((z, y),(w, w))<\varepsilon / 2$. Then $\bar{d}((z, z),(w, w))<\varepsilon$. That is, for every $i>m, g \mid D_{i} \subset U$. It follows that the neighborhood $U$ of $r$ contains the graph of the continuous function $g: I^{n} \rightarrow I^{n}$, and this shows $r$ is almost continuous.

According to [9], we have the following result.

Corollary 3. Let $M$ be the continuum in Theorem 3. Then $M$ has the fixed point property.

\section{REFERENCES}

1. B. D. Garrett, Almost continuity, preprint.

2. S. K. Hildebrand and D. E. Sanderson, Connectivity functions and retracts, Fund. Math. 57 (1965), 237-245.

3. J. H. V. Hunt, A connectivity map $f: S^{n} \rightarrow S^{n-1}$ does not commute with the antipodal map, Bol. Soc. Mat. Mexicana (2) 16 (1971), 43-45.

4. K. R. Kellum, An almost continuous function $f: S^{n} \rightarrow S^{m}$ which commutes with the antipodal map, Proc. Amer. Math. Soc. 54 (1976), 431-432.

5. $135-140$.

6. The equivalence of absolute almost continuous retracts and $\varepsilon$-absolute retracts, Fund. Math. 96 (1977), 229-235.

7. H. Rosen, Connected projections of blocking sets of $I^{n} \times M$, Proc. Amer. Math. Soc. 79 (1980), 335-337.

8. C. P. Rourke and B. J. Sanderson, Introduction to piecewise-linear topology, Springer-Verlag, Berlin and New York, 1972.

9. J. Stallings, Fixed point theorems for connectivity maps, Fund. Math. 47 (1959), 249-263.

Department of Mathematics, University of Alabama, University, Alabama 35486 